\section{Robust Fault Detection for Networked Systems with Distributed Sensors}

\author{
XIAO HE \\ Tsinghua University
}

ZIDONG WANG, Senior Member, IEEE

Brunel University

Y. D. JI

D. H. ZHOU, Senior Member, IEEE

Tsinghua University

This paper is concerned with the robust fault detection problem for a class of discrete-time networked systems with distributed sensors. Since the bandwidth of the communication channel is limited, packets from different sensors may be dropped with different missing rates during the transmission. Therefore, a diagonal matrix is introduced to describe the multiple packet dropout phenomenon and the parameter uncertainties are supposed to reside in a polytope. The aim is to design a robust fault detection filter such that, for all probabilistic packet dropouts, all unknown inputs and admissible uncertain parameters, the error between the residual (generated by the fault detection filter) and the fault signal is made as small as possible. Two parameter-dependent approaches are proposed to obtain less conservative results. The existence of the desired fault detection filter can be determined from the feasibility of a set of linear matrix inequalities that can be easily solved by the efficient convex optimization method. A simulation example on a networked three-tank system is provided to illustrate the effectiveness and applicability of the proposed techniques.

Manuscript received December 27, 2007; revised April 9, 2009; released for publication August 1, 2009.

IEEE Log No. T-AES/47/1/940022.

Refereeing of this contribution was handled by M. Blanke.

This work was supported by national 973 project under Grants 2009CB320602 and 2010CB731800, and the NSFC under Grants 60721003 and 60736026.

Authors' addresses: X. He, Y. D. Ji, and D. H. Zhou, Dept. of Automation, Tsinghua University, Beijing 100084, P.R. China, E-mail: (thuxiao@tsinghua.edu.cn); Z. Wang, Dept. of Information Systems and Computing, Brunel University, Uxbridge, Middlesex, UB8 3PH, UK.

0018-9251/11/\$26.00 (C) 2011 IEEE

\section{INTRODUCTION}

Due to the rapid development of network technology, more and more practical complex systems have their sensors, actuators, and controllers connected via communication networks $[3,14$, $27,32]$. These kinds of systems, also called networked control systems (NCSs), can be found in a variety of engineering areas such as automobiles, manufacturing plants, and aircrafts systems. With respect to traditional control systems, NCSs have many advantages such as low cost, reduced weight and power requirements, simple installation and maintenance, and high reliability. However, the usage of network cables inevitably makes the analysis and design of the NCSs complicated. The limited bandwidth of the communication channel brings new issues such as network-induced time delay, data missing, and quantization effect, which constitute potential sources of instability and poor performance of NCSs. Methods developed from conventional control theory are no longer applicable for the analysis and design for NCSs and should be modified to account for the additional complexity $[1,21]$. Among the effects introduced by the limited bandwidth of communication networks, in this paper, attention is focused on the data missing phenomenon that results from transmission errors in physical network links or from buffer overflows due to congestion $[8,10]$.

Packet dropouts can be modeled within a deterministic framework [29, 30, 32]. However, more and more research attention has recently been paid to various probabilistic settings since the packet dropout is inherently of a random bahavior. For example, in [22], [23], a Bernoulli distributed white sequence taking on values of 0 and 1 has been used to describe the missing measurement. In [18], finite-state Markov chains have been introduced to model correlated dropouts. In [25], Poisson processes have been employed to model stochastic dropouts in continuous time. Very recently, the multiple packet dropouts have been considered in [19], [20] where the number of possible consecutive packet dropouts is limited by a known bound.

It is worth pointing out that, in the context of network-induced packet dropouts, most existing literature has dealt with the problems of stability analysis, controller synthesis, or the state estimation $[6,26]$. Although the fault detection and isolation (FDI) is widely recognized as an important technique to guarantee higher safety and reliability standards of modern control systems [4, 16], only few results have been dedicated to the topic of FDI for networked systems [5, 31]. On the other hand, most reported results concerning NCSs have focused on the case when the network has one channel only [28] or the case when the measurements at a certain time instant 
are sent via the communication network in one packet. However, in many real networked systems, it is quite common that the sensors are spatially located in the remote node $[11,12,15]$, and data from different sensors may have different missing rates (dropout probabilities) for different channels due to their individual characteristics. Furthermore, modeling errors are generally unavoidable and any system design should be robust against possible parameter uncertainties or variations. Unfortunately, up to now, the robust fault detection problem has not yet drawn any research attention for networked systems with distributed sensors having individual data missing probability, and the purpose of this paper is therefore to shorten such a gap.

In this paper, attention is focused on a kind of networked systems with distributed sensors as shown in Fig. 1. At a certain time instant, data from different sensors are packed in different packets and then sent to the fault detection node via communication networks. Packets are transmitted through different channels, and they may be lost at different missing rates due to the limited bandwidth of the common communication channels. A diagonal matrix is introduced to model the multiple packet dropout phenomenon, and polytopic type uncertainties of system parameters are considered. Two parameter-dependent approaches are developed to reduce the design conservatism from traditional quadratic stability methods.

Notation: The notations used throughout the paper are fairly standard. $\mathbb{R}^{n}$ and $\mathbb{R}^{n \times m}$ denote, respectively, the $n$ dimensional Euclidean space and the set of all $n \times m$ real matrices; $A^{T}$ denotes the transpose of a matrix $A ; P>0$ means that $P$ is real symmetric and positive definite; $\operatorname{Pr}\{\iota\}$ represents the occurrence probability of the event "ı"; $\mathbb{E}\{\varrho\}$ stands for the expectation of a stochastic variable $\varrho$; $l_{2}[0, \infty)$ is the space of all square-summable vector functions over $[0, \infty) ;\|x\|$ is the standard $l_{2}$ norm of $x$, i.e., $\|x\|=\left(x^{T} x\right)^{1 / 2}$. In symmetric block matrices, “*” is introduced to represent the term that can be determined by symmetry. $\operatorname{diag}\{\cdots\}$ stands for a block-diagonal matrix and the notation $\operatorname{diag}_{q}\{\xi\}$ is employed to represent $\operatorname{diag}\{\underbrace{\xi \cdots \xi}_{q}\}$.

\section{PROBLEM FORMULATION AND PRELIMINARIES}

Consider the following class of discrete-time networked systems with distributed sensors:

$$
\begin{aligned}
x_{k+1} & =A_{\lambda} x_{k}+B_{\lambda} w_{k}+E_{\lambda} f_{k} \\
x_{0} & =\varphi_{0}
\end{aligned}
$$

where $x_{k} \in \mathbb{R}^{n}$ is the state vector; $w_{k} \in \mathbb{R}^{p}$ is the disturbance input belonging to $l_{2}[0, \infty) ; f_{k} \in \mathbb{R}^{q}$ is the fault to be detected; $\varphi_{0}$ is a given initial value; $A_{\lambda}$,

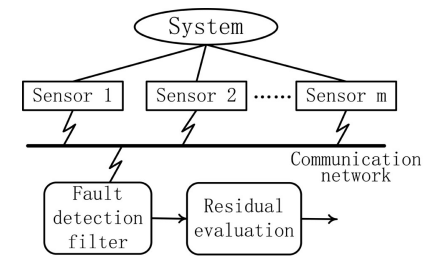

Fig. 1. Fault detection for networked system with distributed sensors.

$B_{\lambda}$, and $E_{\lambda}$ are real constant matrices with appropriate dimensions.

Since the bandwidth of the communication channel is limited, data generated from sensors may be lost or occupied by noise during the transmission process. Consider that the sensors are distributively located at the system side, packets from different sensors may be lost with different missing rates. To describe this phenomenon, a diagonal matrix $\Theta_{k}$ is introduced which results in the following new measurement model:

$$
y_{k}=\Theta_{k} C_{\lambda} x_{k}+D_{\lambda} w_{k}
$$

where $y_{k} \in \mathbb{R}^{m}$ is the measurement received at the fault detection filter node. $\Theta_{k}=\operatorname{diag}\left\{\theta_{k}^{1}, \ldots, \theta_{k}^{m}\right\}$ is a diagonal matrix that accounts for the different missing rate of the individual channel. Specifically, for any $1 \leq r \leq m, \theta_{k}^{r} \in\{0,1\}$ is a Bernoulli distributed stochastic variable satisfying

$$
\begin{aligned}
& \operatorname{Pr}\left\{\theta_{k}^{r}=1\right\}=\mathbb{E}\left\{\theta_{k}^{r}\right\}=\beta^{r} \\
& \operatorname{Pr}\left\{\theta_{k}^{r}=0\right\}=1-\mathbb{E}\left\{\theta_{k}^{r}\right\}=1-\beta^{r} .
\end{aligned}
$$

where $\beta^{r}(1 \leq r \leq m)$ are exactly known scalars taking values in $[0,1]$.

REMARK 1 Similar to the idea of incompleteness matrix in measurement model adopted in [17], a diagonal matrix with its diagonal entries taking values of 0 or 1 is employed to model the multiple packets dropout phenomenon in networked system with distributed sensors. $\theta_{k}^{r}=0$ corresponds the dropout of the $r$ th packet in time instant $k$ and $\theta_{k}^{r}=1$ represents the ideal transmission of this packet. In terms of such a newly introduced diagonal matrix, the following three cases that usually occur in a networked environment can be dealt with in a unified framework. a) The first is ideal transmission, which means that the network status is perfect and no packet dropout phenomenon happens. In this case, $\beta^{r}=1$ for $1 \leq r \leq m$. b) The second partial packet dropouts, where some packets are lost during the transmission and, at a certain time, only part of the measurements are received by the fault detection filter node. This corresponds to the multiple packet case with $\beta^{r}=0$ for some $1 \leq r \leq m$. c) The third is one packet transmission case, where at a certain instant, all dimensions of the measurement are either lost completely or transmitted successfully with the same missing rate. This binary measurement model can be 
recovered by imposing $\theta_{k}^{r}=\theta_{k}$ for all $1 \leq r \leq m$ and is widely used in recent literature [22, 23].

The above measurement model (2) can be further transformed into the following form

$$
y_{k}=\sum_{r=1}^{m} \theta_{k}^{r} C_{\lambda}^{r} x_{k}+D_{\lambda} w_{k}
$$

where

$$
C_{\lambda}^{r}=\operatorname{diag}\{\overbrace{0, \ldots, 0,0}^{r-1}, 1, \overbrace{0, \ldots, 0}^{m-r}\} C_{\lambda} .
$$

All system matrices in (1) and (2) are supposed to have appropriate dimensions. Note that in practice it may be difficult or impossible to obtain exact models. Therefore, the present paper considers the parameters which are subject to uncertainties of polytopic type, i.e.,

$$
\Omega_{\lambda}=\left(A_{\lambda}, B_{\lambda}, E_{\lambda}, C_{\lambda}, D_{\lambda}\right) \in \Re
$$

where $\Re$ is a given convex polyhedral domain described by $v$ vertices:

$$
\Re=\left\{\Omega_{\lambda} \mid \Omega_{\lambda}=\sum_{i=1}^{v} \lambda_{i} \Omega_{i} ; \sum_{i=1}^{v} \lambda_{i}=1, \lambda_{i} \geq 0\right\}
$$

and $\Omega_{i}=\left(A_{i}, B_{i}, E_{i}, C_{i}, D_{i}\right)$ denotes the $i$ th vertex of the polytope.

A full-order fault detection filter of the following form is interested in

$$
\begin{aligned}
\tilde{x}_{k+1} & =G \tilde{x}_{k}+K y_{k} \\
r_{k} & =L \tilde{x}_{k}
\end{aligned}
$$

where $\tilde{x}_{k} \in \mathbb{R}^{n}$ is the filter state vector, $r_{k} \in \mathbb{R}^{q}$ is the so-called residual that is compatible with the fault vector $f_{k}$. Our main aim is to make the error between residual and fault signal as small as possible.

By defining $\eta_{k}=\left[\begin{array}{ll}x_{k}^{T} & \tilde{x}_{k}^{T}\end{array}\right]^{T}, v_{k}=\left[\begin{array}{ll}w_{k}^{T} & f_{k}^{T}\end{array}\right]^{T}$ and $\tilde{r}_{k}=r_{k}-f_{k}$, we obtain the following overall fault detection dynamics.

$$
\begin{aligned}
\eta_{k+1} & =\left[\mathcal{A}_{\lambda}^{0}+\sum_{r=1}^{m}\left(\theta_{k}^{r}-\beta^{r}\right) \mathcal{A}_{\lambda}^{r}\right] \eta_{k}+\mathcal{B}_{\lambda} v_{k} \\
\tilde{r}_{k} & =\mathcal{C}_{\lambda} \eta_{k}+\mathcal{D}_{\lambda} v_{k}
\end{aligned}
$$

with initial condition $\eta_{0}$, where

$$
\begin{aligned}
& \mathcal{A}_{\lambda}^{0}=\left[\begin{array}{cc}
A_{\lambda} & 0 \\
\sum_{r=1}^{m} \beta^{r} K C_{\lambda}^{r} & G
\end{array}\right] \\
& \mathcal{A}_{\lambda}^{r}=\left[\begin{array}{cc}
0 & 0 \\
K C_{\lambda}^{r} & 0
\end{array}\right], \quad \mathcal{B}_{\lambda}=\left[\begin{array}{cc}
B_{\lambda} & E_{\lambda} \\
K D_{\lambda} & 0
\end{array}\right] \\
& \mathcal{C}_{\lambda}=\left[\begin{array}{ll}
0 & L
\end{array}\right], \quad \mathcal{D}_{\lambda}=\left[\begin{array}{ll}
0 & -I
\end{array}\right] .
\end{aligned}
$$

Considering the existence of the stochastic variables $\theta_{k}^{r}(1 \leq r \leq m)$, let us recall the definition of stochastic stability in the mean-square sense for the fault detection system (9).
DEFINITION 1 Reference [22] system (9) with $v_{k}=0$ is said to be exponentially mean-square stable if, for any initial conditions, there exist constants $\alpha>0$ and $\kappa \in(0,1)$ such that

$$
\mathbb{E}\left\{\left\|\eta_{k}\right\|^{2}\right\} \leq \alpha \kappa^{k} \mathbb{E}\left\{\left\|\eta_{0}\right\|^{2}\right\}, \quad k \in \mathbb{Z}^{+} .
$$

Assumption 1 System (1) is exponentially mean-square stable or has been stabilized by local controller (or networked controller) to be exponentially mean-square stable.

Based on the above definition, the robust network-based fault detection filter design (RNFDFD) problem with distributed sensors can now be formulated as the following robust $H_{\infty}$ filtering problem $[7,9,26]$ : designing a robust $H_{\infty}$ filter of the form (8) for the system (1)-(2) such that, for all admissible parameter uncertainties and possible multiple packet dropouts, 1) the overall fault detection dynamics (9) are exponentially mean-square stable when $v_{k}=0$ and, 2) under zero initial condition, the infimum of $\gamma$ is made small in the feasibility of

$$
\sup _{v_{k} \neq 0} \frac{\mathbb{E}\left\{\left\|\tilde{r}_{k}\right\|^{2}\right\}}{\left\|v_{k}\right\|^{2}}<\gamma^{2}, \quad \gamma>0
$$

In this paper, the residual evaluation function $J_{k}$ as well as the threshold $J_{t h}$ are adopted as

$$
\begin{aligned}
J_{k} & =\left\{\sum_{s=0}^{k} r_{s}^{T} r_{s}\right\}^{1 / 2} \\
J_{t h} & =\sup _{v_{k} \in l_{2}, f_{k}=0} \mathbb{E}\left\{J_{k}\right\} .
\end{aligned}
$$

Furthermore, the occurrence of faults can then be detected by comparing $J_{k}$ with the prescribed $J_{t h}$ according to:

$$
\begin{aligned}
& J_{k}>J_{t h} \Rightarrow \text { with faults } \Rightarrow \text { alarm } \\
& J_{k} \leq J_{t h} \Rightarrow \text { no faults. }
\end{aligned}
$$

\section{FAULT DETECTION FILTER PERFORMANCE ANALYSIS}

In this section, for a given fault detection filter, the $H_{\infty}$ performance analysis problem is discussed. The following lemma is helpful in deriving our main results in the sequel.

LEMMA 1 Consider the networked system (1) with multiple packet dropouts (2). Given a scalar $\gamma>0$ and a fault detection filter (8) with parameters $G, K$ and $L$, if there exists a positive definite matrix $P_{\lambda}=P_{\lambda}^{T}>0$ satisfying

$$
\left[\begin{array}{ccccc}
-I & 0 & 0 & \mathcal{C}_{\lambda} & \mathcal{D}_{\lambda} \\
* & -P_{\lambda d} & 0 & \rho_{d} P_{\lambda d} \mathcal{A}_{\lambda}^{c} & 0 \\
* & * & -P_{\lambda} & P_{\lambda} \mathcal{A}_{\lambda}^{0} & P_{\lambda} \mathcal{B}_{\lambda} \\
* & * & * & -P_{\lambda} & 0 \\
* & * & * & * & -\gamma^{2} I
\end{array}\right]<0
$$


where $P_{\lambda d}=\operatorname{diag}_{m}\left\{P_{\lambda}\right\}, \rho_{d}=\operatorname{diag}\left\{\rho_{1} I_{2 n}, \ldots, \rho_{m} I_{2 n}\right\}$, $\rho_{r}=\sqrt{\beta^{r}\left(1-\beta^{r}\right)}(1 \leq r \leq m), \mathcal{A}_{\lambda}^{c}=\left[\mathcal{A}_{\lambda}^{1 T} \ldots \mathcal{A}_{\lambda}^{m T}\right]^{T}$, and $\mathcal{A}_{\lambda}^{0}, \mathcal{A}_{\lambda}^{r}, \mathcal{B}_{\lambda}, \mathcal{C}_{\lambda}, \mathcal{D}_{\lambda}$ is defined in (10), then the fault detection dynamic (9) is exponentially mean-square stable and satisfies the $H_{\infty}$ attenuation condition (12).

PROOF Consider a Lyapunov functional

$$
V_{k}=\eta_{k}^{T} P_{\lambda} \eta_{k}
$$

where $P_{\lambda}>0$. Noting that for $1 \leq r \leq m$ and $1 \leq s \leq m$,

$$
\mathbb{E}\left\{\left(\theta_{k}^{r}-\beta^{r}\right)\left(\theta_{k}^{s}-\beta^{s}\right)\right\}= \begin{cases}\beta^{r}\left(1-\beta^{r}\right), & r=s \\ 0, & r \neq s\end{cases}
$$

we can calculate the difference of $V_{k}$ with $v_{k}=0$ as below:

$$
\begin{aligned}
\Delta V_{k} & :=\mathbb{E}\left\{V_{k+1}\left(\eta_{k+1}\right) \mid \eta_{k}, \ldots, \eta_{0}\right\}-V_{k}\left(\eta_{k}\right) \\
& =\eta_{k}^{T}\left[\mathcal{A}_{\lambda}^{0 T} P_{\lambda} \mathcal{A}_{\lambda}^{0}+\sum_{r=1}^{m} \rho_{r}^{2} \mathcal{A}_{\lambda}^{r T} P_{\lambda} \mathcal{A}_{\lambda}^{r}-P_{\lambda}\right] \eta_{k} .
\end{aligned}
$$

It follows from Schur complement [2] that (LMI) (14) implies $\Delta V_{k}<0$ for all non-zero $\eta_{k}$. A proper positive scalar $\vartheta>0$ can also be found such that $\Delta V_{k}<-\vartheta\left\|\eta_{k}\right\|^{2}$. Furthermore, from [22, Lemma 1], it can be confirmed that the fault detection system (9) is exponentially mean-square stable.

Next, for any non-zero $v_{k}$, it follows from (9) and (15) that

$$
\Delta V_{k}+\mathbb{E}\left\{\tilde{r}_{k}^{T} \tilde{r}_{k}\right\}-\gamma^{2} \mathbb{E}\left\{v_{k}^{T} v_{k}\right\} \leq \chi_{k}^{T}\left[\begin{array}{cc}
\Xi_{1} & \Xi_{2} \\
* & \Xi_{3}
\end{array}\right] \chi_{k}
$$

where

$$
\begin{aligned}
& \chi_{k}=\left[\begin{array}{ll}
\eta_{k}^{T} & v_{k}^{T}
\end{array}\right]^{T} \\
& \Xi_{1}=\mathcal{A}_{\lambda}^{0 T} P_{\lambda} \mathcal{A}_{\lambda}^{0}+\sum_{r=1}^{m} \rho_{r}^{2} \mathcal{A}_{\lambda}^{r T} P_{\lambda} \mathcal{A}_{\lambda}^{r}-P_{\lambda}+\mathcal{C}_{\lambda}^{T} \mathcal{C}_{\lambda} \\
& \Xi_{2}=\mathcal{A}_{\lambda}^{0 T} P_{\lambda} \mathcal{B}_{\lambda}+\mathcal{C}_{\lambda}^{T} \mathcal{D}_{\lambda} \\
& \Xi_{3}=\mathcal{B}_{\lambda}^{T} P_{\lambda} \mathcal{B}_{\lambda}+\mathcal{D}_{\lambda}^{T} \mathcal{D}_{\lambda}-\gamma^{2} I .
\end{aligned}
$$

Again, using Schur complement [2], it can be observed from (14) and (17) that for any $\eta_{k}$ and $v_{k}$ that are not all zero,

$$
\Delta V_{k}+\mathbb{E}\left\{\tilde{r}_{k}^{T} \tilde{r}_{k}\right\}-\gamma^{2} \mathbb{E}\left\{v_{k}^{T} v_{k}\right\}<0 .
$$

Now, summing up (18) from 0 to $\infty$ with respect to $k$ yields

$$
\sum_{k=0}^{\infty} \mathbb{E}\left\{\left\|\tilde{r}_{k}\right\|^{2}\right\}<\gamma^{2} \sum_{k=0}^{\infty} \mathbb{E}\left\{\left\|v_{k}\right\|^{2}\right\}+\mathbb{E}\left\{V_{0}\right\}-\mathbb{E}\left\{V_{\infty}\right\} .
$$

Since the system (9) is exponentially mean-square stable, it is straightforward to see that (12) holds under the zero initial condition. The proof is completed.

\section{ROBUST FAULT DETECTION FILTER DESIGN}

This section provides a solution to the RNFDFD design problem. The following is an equivalent form of Lemma 1.

LEMMA 2 Consider the networked system (1) with distributed sensors (2) and a given fault detection filter of the form (8). For any fixed $\Omega_{\lambda} \in \Re$, there exists a matrix $P_{\lambda}=P_{\lambda}^{T}>0$ such that (14) holds if and only if there exist matrices $P_{\lambda}=P_{\lambda}^{T}>0$ and $M_{\lambda}$ satisfying

$$
\left[\begin{array}{ccccc}
-I & 0 & 0 & \mathcal{C}_{\lambda} & \mathcal{D}_{\lambda} \\
* & \Psi_{1} & 0 & \rho_{d} M_{\lambda d}^{T} \mathcal{A}_{\lambda}^{c} & 0 \\
* & * & \Psi_{2} & M_{\lambda}^{T} \mathcal{A}_{\lambda}^{0} & M_{\lambda}^{T} \mathcal{B}_{\lambda} \\
* & * & * & -P_{\lambda} & 0 \\
* & * & * & * & -\gamma^{2} I
\end{array}\right]<0
$$

where $\Psi_{1}=P_{\lambda d}-M_{\lambda d}^{T}-M_{\lambda d}, \Psi_{2}=P_{\lambda}-M_{\lambda}^{T}-M_{\lambda}$, $M_{\lambda d}=\operatorname{diag}_{m}\left\{M_{\lambda}\right\}$.

PROOF The proof is similar with that for Corollary 1 in [9] and is omitted here.

REMARK 2 The superiority of Lemma 2 to Lemma 1 owes to the introduction of an additional free matrix $M_{\lambda}$, which eliminates the product term of $P_{\lambda}$ and the system matrices. This provides us an effective way to get a less conservative than the "quadratic result" [9] when parameter uncertainty is taken into consideration in the robust fault detection design procedure.

The following theorem deals with the addressed fault detection filter design problem with fixed system parameters.

THEOREM 1 Consider the networked system (1) and (2) with fixed and known parameters $\Omega_{\lambda} \in \Re$. There exist matrices $P_{\lambda}=P_{\lambda}^{T}>0, M_{\lambda}$, as well as a filter with parameters $G, K, L$ satisfying (20) if and only if there exist $\bar{P}_{\lambda}>0, R_{\lambda}, S_{\lambda}, T_{\lambda}, \bar{G}, \bar{K}, \bar{L}$, satisfying

$$
\left[\begin{array}{ccccc}
-I & 0 & 0 & \Phi_{1} & \Phi_{2} \\
* & \Phi_{3} & 0 & \Phi_{4} & 0 \\
* & * & \Phi_{5} & \Phi_{6} & \Phi_{7} \\
* & * & * & \Phi_{8} & 0 \\
* & * & * & * & -\gamma^{2} I
\end{array}\right]<0
$$

where $\tilde{\rho}_{d}=\operatorname{diag}\left\{\rho_{1} I_{n}, \ldots, \rho_{m} I_{n}\right\}$ and

$$
\begin{aligned}
\Phi_{1} & =\left[\begin{array}{ll}
0 & \bar{L}
\end{array}\right], \quad \Phi_{2}=\left[\begin{array}{ll}
0 & -I
\end{array}\right] \\
\Phi_{3} & =\left[\begin{array}{cc}
\bar{P}_{1 \lambda d}-R_{\lambda d}-R_{\lambda d}^{T} & \bar{P}_{2 \lambda d}-S_{\lambda d}-T_{\lambda d} \\
* & \bar{P}_{3 \lambda d}-T_{\lambda d}-T_{\lambda d}^{T}
\end{array}\right]
\end{aligned}
$$




$$
\begin{aligned}
& \Phi_{4}= {\left[\begin{array}{ll}
\tilde{\rho}_{d} \bar{K}_{d} C_{\lambda}^{c} & 0 \\
\tilde{\rho}_{d} \bar{K}_{d} C_{\lambda}^{c} & 0
\end{array}\right], \quad \bar{K}_{d}=\operatorname{diag}_{m}\{\bar{K}\} } \\
& \Phi_{5}=\left[\begin{array}{cc}
\bar{P}_{1 \lambda}-R_{\lambda}-R_{\lambda}^{T} & \bar{P}_{2 \lambda}-S_{\lambda}-T_{\lambda} \\
* & \bar{P}_{3 \lambda}-T_{\lambda}-T_{\lambda}^{T}
\end{array}\right] \\
& \Phi_{6}=\left[\begin{array}{cc}
R_{\lambda}^{T} A_{\lambda}+\bar{K} \sum_{r=1}^{m} \beta^{r} C_{\lambda}^{r} & \bar{G} \\
S_{\lambda}^{T} A_{\lambda}+\bar{K} \sum_{r=1}^{m} \beta^{r} C_{\lambda}^{r} & \bar{G}
\end{array}\right] \\
& \Phi_{7}=\left[\begin{array}{cc}
R_{\lambda}^{T} B_{\lambda}+\bar{K} D_{\lambda} & R_{\lambda}^{T} E_{\lambda} \\
S_{\lambda}^{T} B_{\lambda}+\bar{K} D_{\lambda} & S_{\lambda}^{T} E_{\lambda}
\end{array}\right] \\
& \Phi_{8}=-\bar{P}_{\lambda}, \quad \bar{P}_{\lambda}=\left[\begin{array}{cc}
\bar{P}_{1 \lambda} & \bar{P}_{2 \lambda} \\
* & \bar{P}_{3 \lambda}
\end{array}\right] \\
& \bar{P}_{1 \lambda d}=\operatorname{diag}_{m}\left\{\bar{P}_{1 \lambda}\right\}, \quad \bar{P}_{2 \lambda d}=\operatorname{diag}_{m}\left\{\bar{P}_{2 \lambda}\right\} \\
& \bar{P}_{3 \lambda d}=\operatorname{diag}_{m}\left\{\bar{P}_{3 \lambda}\right\}, \quad R_{\lambda d}=\operatorname{diag}_{m}\left\{R_{\lambda}\right\} \\
& \mathrm{S}_{\lambda d}=\operatorname{diag}_{m}\left\{S_{\lambda}\right\}, \quad T_{\lambda d}=\operatorname{diag}_{m}\left\{T_{\lambda}\right\} .
\end{aligned}
$$

Moreover, if (21) is feasible, the parameters of the desired fault detection filter can be obtained by

$$
\left[\begin{array}{cc}
G & K \\
L & 0
\end{array}\right]=\left[\begin{array}{cc}
T_{\lambda}^{-1} & 0 \\
0 & I
\end{array}\right]\left[\begin{array}{cc}
\bar{G} & \bar{K} \\
\bar{L} & 0
\end{array}\right] .
$$

Proof (Necessity) Suppose there exist matrices $P_{\lambda}, M_{\lambda}$ and fault detection filter parameters $G, K, L$ satisfying (20). Rewrite the following matrices into the block type of the following form

$$
P_{\lambda}=\left[\begin{array}{cc}
P_{1 \lambda} & P_{2 \lambda} \\
* & P_{3 \lambda}
\end{array}\right], \quad M_{\lambda}=\left[\begin{array}{ll}
M_{1 \lambda} & M_{2 \lambda} \\
M_{3 \lambda} & M_{4 \lambda}
\end{array}\right] .
$$

Without loss of generality, it can be assumed that $M_{3 \lambda}$ and $M_{4 \lambda}$ are nonsingular. Define

$$
\begin{aligned}
\mathcal{T}_{\lambda} & =\left[\begin{array}{cc}
I & 0 \\
0 & M_{4 \lambda}^{-1} M_{3 \lambda}
\end{array}\right], \\
\mathcal{T}_{\lambda d} & =\operatorname{diag}_{m}\left\{\mathcal{T}_{\lambda}\right\} \\
\bar{P}_{\lambda} & =\left[\begin{array}{cc}
\bar{P}_{1 \lambda} & \bar{P}_{2 \lambda} \\
* & \bar{P}_{3 \lambda}
\end{array}\right]=\mathcal{T}_{\lambda}^{T} P_{\lambda} \mathcal{T}_{\lambda} .
\end{aligned}
$$

Postmultiplying and premultiplying (20) with $\operatorname{diag}\left\{I, \mathcal{T}_{\lambda d}, \mathcal{T}_{\lambda}, I, I\right\}$ and its transpose, together with (10), it can be obtained that

$$
\left[\begin{array}{ccccc}
-I & 0 & 0 & \bar{\Phi}_{1} & \mathcal{D}_{\lambda} \\
* & \operatorname{diag}_{m}\left\{\bar{\Phi}_{3}\right\} & 0 & \bar{\Phi}_{2} & 0 \\
* & * & \bar{\Phi}_{3} & \bar{\Phi}_{4} & \bar{\Phi}_{5} \\
* & * & * & -\bar{P}_{\lambda} & 0 \\
* & * & * & * & -\gamma^{2} I
\end{array}\right]<0
$$

where

$$
\begin{aligned}
& \bar{\Phi}_{1}=\left[\begin{array}{ll}
0 & L M_{4 \lambda}^{-1} M_{3 \lambda}
\end{array}\right], \quad \bar{\Phi}_{3}=\left[\begin{array}{cc}
\bar{\Phi}_{6} & \bar{\Phi}_{7} \\
* & \bar{\Phi}_{8}
\end{array}\right] \\
& \bar{\Phi}_{2}=\left[\begin{array}{ll}
{\left[\begin{array}{ll}
\rho_{1} M_{3 \lambda}^{T} K C_{\lambda}^{1} & 0 \\
\rho_{1} M_{3 \lambda}^{T} K C_{\lambda}^{1} & 0
\end{array}\right]^{T}, \ldots,\left[\begin{array}{cc}
\rho_{m} M_{3 \lambda}^{T} K C_{\lambda}^{m} & 0 \\
\rho_{m} M_{3 \lambda}^{T} K C_{\lambda}^{m} & 0
\end{array}\right]^{T}}
\end{array}\right]^{T} \\
& \bar{\Phi} 4=\left[\begin{array}{cc}
M_{1 \lambda}^{T} A_{\lambda}+\bar{\Phi}_{9} & M_{3 \lambda}^{T} G M_{4 \lambda}^{-1} M_{3 \lambda} \\
M_{3 \lambda}^{T} M_{4 \lambda}^{-T} M_{2 \lambda}^{T} A_{\lambda}+\bar{\Phi}_{9} & M_{3 \lambda}^{T} G M_{4 \lambda}^{-1} M_{3 \lambda}
\end{array}\right] \\
& \bar{\Phi}_{5}=\left[\begin{array}{cc}
M_{1 \lambda}^{T} B_{\lambda}+M_{3 \lambda}^{T} K D_{\lambda} & M_{1 \lambda}^{T} E_{\lambda} \\
M_{3 \lambda}^{T} M_{4 \lambda}^{-T} M_{2 \lambda}^{T} B_{\lambda}+M_{3 \lambda}^{T} K D_{\lambda} & M_{3 \lambda}^{T} M_{4 \lambda}^{-T} M_{2 \lambda}^{T} E_{\lambda}
\end{array}\right] \\
& \bar{\Phi}_{6}=\bar{P}_{1 \lambda}-M_{1 \lambda}-M_{1 \lambda}^{T}, \\
& \bar{\Phi}_{7}=\bar{P}_{2 \lambda}-M_{2 \lambda} M_{4 \lambda}^{-1} M_{3 \lambda}-M_{3 \lambda}^{T} M_{4 \lambda}^{-1} M_{3 \lambda} \\
& \bar{\Phi}_{8}=\bar{P}_{3 \lambda}-M_{3 \lambda}^{T} M_{4 \lambda}^{-1} M_{3 \lambda}-M_{3 \lambda}^{T} M_{4 \lambda}^{-T} M_{3 \lambda} \\
& \bar{\Phi}_{9}=\sum_{r=1}^{m} \beta^{r} M_{3 \lambda}^{T} K C_{\lambda}^{r} .
\end{aligned}
$$

Consider a new matrix $\Lambda \in \mathbb{R}^{2 m n \times 2 m n}$ with its entries being $\Lambda_{\alpha \beta,(2 \alpha-1) \beta}=\Lambda_{(\alpha+m) \beta, 2 \alpha \beta}=1$ for all $1 \leq \alpha \leq m$ and $1 \leq \beta \leq n$, and other entries being all zero. Once again, perform congruence transformation to (24) by $\operatorname{diag}\left\{I, \Lambda^{T}, I, I, I\right\}$, we can infer that (24) is equivalent to

$$
\left[\begin{array}{ccccc}
-I & 0 & 0 & \bar{\Phi}_{1} & \mathcal{D}_{\lambda} \\
* & \tilde{\Phi}_{3} & 0 & \tilde{\Phi}_{2} & 0 \\
* & * & \bar{\Phi}_{3} & \bar{\Phi}_{4} & \bar{\Phi}_{5} \\
* & * & * & -\bar{P}_{\lambda} & 0 \\
* & * & * & * & -\gamma^{2} I
\end{array}\right]<0
$$

where

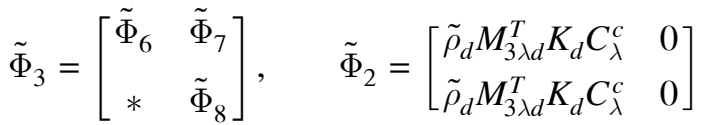

$$
\begin{aligned}
& \tilde{\Phi}_{6}=\bar{P}_{1 \lambda d}-M_{1 \lambda d}-M_{1 \lambda d}^{T}, \quad C_{\lambda}^{c}=\left[C_{\lambda}^{1 T} \ldots C_{\lambda}^{m T}\right]^{T} \\
& \tilde{\Phi}_{7}=\bar{P}_{2 \lambda d}-M_{2 \lambda d} M_{4 \lambda d}^{-1} M_{3 \lambda d}-M_{3 \lambda d}^{T} M_{4 \lambda d}^{-1} M_{3 \lambda d} \\
& \tilde{\Phi}_{8}=\bar{P}_{3 \lambda d}-M_{3 \lambda d}^{T} M_{4 \lambda d}^{-1} M_{3 \lambda d}-M_{3 \lambda d}^{T} M_{4 \lambda d}^{-T} M_{3 \lambda d} \\
& M_{1 \lambda d}=\operatorname{diag}_{m}\left\{M_{1 \lambda}\right\}, \quad M_{2 \lambda d}=\operatorname{diag}_{m}\left\{M_{2 \lambda}\right\} \\
& M_{3 \lambda d}=\operatorname{diag}_{m}\left\{M_{3 \lambda}\right\}, \quad M_{4 \lambda d}=\operatorname{diag}_{m}\left\{M_{4 \lambda}\right\} \\
& K_{d}=\operatorname{diag}_{m}\{K\} .
\end{aligned}
$$

Define new matrices

$$
\begin{aligned}
& R_{\lambda}=M_{1 \lambda}, \quad S_{\lambda}=M_{2 \lambda} M_{4 \lambda}^{-1} M_{3 \lambda} \\
& T_{\lambda}=M_{3 \lambda}^{T} M_{4 \lambda}^{-1} M_{3 \lambda}, \quad \bar{G}=M_{3 \lambda}^{T} G M_{4 \lambda}^{-1} M_{3 \lambda} \\
& \bar{K}=M_{3 \lambda}^{T} K, \quad \bar{L}=L M_{4 \lambda}^{-1} M_{3 \lambda} .
\end{aligned}
$$


By substituting (26) to (25), (21) can be obtained and then the necessity is proved.

(Sufficiency) Suppose there exist matrices $\bar{P}_{\lambda}>0$, $R_{\lambda}, S_{\lambda}, T_{\lambda}, \bar{G}, \bar{K}, \bar{L}$ satisfying (21), it is straightforward to know that $T_{\lambda}$ is nonsingular. It can be further noted that nonsingular matrices $M_{4 \lambda}$ and $M_{3 \lambda}$ satisfy $T_{\lambda}=M_{3 \lambda} M_{4 \lambda}^{-1} M_{3 \lambda}$. Introduce nonsingular matrix $\mathcal{T}_{\lambda}$ as in (23) and define the following matrices

$$
\begin{aligned}
M_{\lambda} & =\left[\begin{array}{cc}
R_{\lambda} & S_{\lambda} M_{3 \lambda}^{-1} M_{4 \lambda} \\
M_{3 \lambda} & M_{4 \lambda}
\end{array}\right] \\
P_{\lambda} & =\mathcal{T}_{\lambda}^{-T} \bar{P}_{\lambda} \mathcal{T}_{\lambda}^{-1} \\
G & =M_{3 \lambda}^{-T} \bar{G} M_{3 \lambda}^{-1} M_{4 \lambda} \\
K & =M_{3 \lambda}^{-T} \bar{K} \\
L & =\bar{L} M_{3 \lambda}^{-1} M_{4 \lambda}
\end{aligned}
$$

one can get $P_{\lambda}>0$.

Performing congruence transformation to (21) first by $\operatorname{diag}\{I, \Lambda, I, I, I\}$ ( $\Lambda$ is defined as before), and second by $\operatorname{diag}\left\{I, \mathcal{T}_{\lambda d}^{-1}, \mathcal{T}_{\lambda}^{-1}, I, I\right\},(20)$ can be obtained. The proof of sufficiency is finished.

Furthermore, from the proof of sufficiency, if there exist matrices $\bar{P}_{\lambda}>0, R_{\lambda}, S_{\lambda}, T_{\lambda}, \bar{G}, \bar{K}, \bar{L}$ satisfying (21), the parameters of the fault detection filter satisfying (20) can be obtained by (27). The transfer function from $v_{k}$ to $\tilde{r}_{k}$ can be further given by

$$
T_{v \tilde{r}}(z)=L(z I-G)^{-1} K .
$$

Substituting (27) into (28) and considering the relationship $T_{\lambda}=M_{3 \lambda}^{T} M_{4 \lambda}^{-1} G_{3 \lambda}$, we can get

$$
\begin{aligned}
T_{v \tilde{r}}(z) & =\bar{L} M_{3 \lambda}^{-1} M_{4 \lambda}\left(z I-M_{3 \lambda}^{-T} \bar{G} M_{3 \lambda}^{-1} M_{4 \lambda}\right)^{-1} M_{3 \lambda}^{-T} \bar{K} \\
& =\bar{L}\left(z I-T_{\lambda}^{-1} \bar{G}\right)^{-1} T_{\lambda}^{-1} \bar{K}
\end{aligned}
$$

which means that the desired fault detection filter parameters can also be given by (22). This ends the proof.

From Theorem 1, we provide two different fault detection filter design results for the networked system (1) with multiple packet dropout (2), both of which are based on the idea of parameter-dependent stability.

THEOREM 2 Consider networked system (1) with parameter uncertainty (6) and multiple packet dropouts (2). The RNFDFD problem is solvable if there exist matrices $\bar{P}_{i}=\bar{P}_{i}^{T}>0, R, S, T, \bar{G}, \bar{K}, \bar{L}$ satisfying

$$
\left[\begin{array}{ccccc}
-I & 0 & 0 & \Psi_{1} & \Psi_{2} \\
* & \Psi_{3} & 0 & \Psi_{4} & 0 \\
* & * & \Psi_{5} & \Psi_{6} & \Psi_{7} \\
* & * & * & \Psi_{8} & 0 \\
* & * & * & * & -\gamma^{2} I
\end{array}\right]<0
$$

for all $1 \leq i \leq v$, where $\tilde{\rho}_{d}=\operatorname{diag}\left\{\rho_{1} I_{n}, \ldots, \rho_{m} I_{n}\right\}$ and

$$
\begin{aligned}
& \Psi_{1}=\left[\begin{array}{ll}
0 & \bar{L}
\end{array}\right], \quad \Psi_{2}=\left[\begin{array}{ll}
0 & -I
\end{array}\right] \\
& \Psi_{3}=\left[\begin{array}{cc}
\bar{P}_{1 i d}-R_{d}-R_{d}^{T} & \bar{P}_{2 i d}-S_{d}-T_{d} \\
* & \bar{P}_{3 i d}-T_{d}-T_{d}^{T}
\end{array}\right] \\
& \Psi_{4}=\left[\begin{array}{cc}
\tilde{\rho}_{d} \bar{K}_{d} C_{i}^{c} & 0 \\
\tilde{\rho}_{d} \bar{K}_{d} C_{i}^{c} & 0
\end{array}\right], \quad \bar{K}_{d}=\operatorname{diag}_{m}\{\bar{K}\} \\
& \Psi_{5}=\left[\begin{array}{cc}
\bar{P}_{1 i}-R-R^{T} & \bar{P}_{2 i}-S-T \\
* & \bar{P}_{3 i}-T-T^{T}
\end{array}\right] \\
& \Psi_{6}=\left[\begin{array}{cc}
R^{T} A_{i}+\bar{K} \sum_{r=1}^{m} \beta^{r} C_{i}^{r} & \bar{G} \\
S^{T} A_{i}+\bar{K} \sum_{r=1}^{m} \beta^{r} C_{i}^{r} & \bar{G}
\end{array}\right] \\
& \Psi_{7}=\left[\begin{array}{cc}
R^{T} B_{i}+\bar{K} D_{i} & R^{T} E_{i} \\
S^{T} B_{i}+\bar{K} D_{i} & S^{T} E_{i}
\end{array}\right] \\
& \Psi_{8}=-\bar{P}_{i}, \quad \bar{P}_{i}=\left[\begin{array}{cc}
\bar{P}_{1 i} & \bar{P}_{2 i} \\
* & \bar{P}_{3 i}
\end{array}\right] \\
& \bar{P}_{1 i d}=\operatorname{diag}_{m}\left\{\bar{P}_{1 i}\right\}, \quad \bar{P}_{2 i d}=\operatorname{diag}_{m}\left\{\bar{P}_{2 i}\right\} \\
& \bar{P}_{3 i d}=\operatorname{diag}_{m}\left\{\bar{P}_{3 i}\right\}, \quad R_{d}=\operatorname{diag}_{m}\{R\} \\
& S_{d}=\operatorname{diag}_{m}\{S\}, \quad T_{d}=\operatorname{diag}_{m}\{T\} .
\end{aligned}
$$

Moreover, if (30) is feasible, the parameters of the desired fault detection filter can be given by

$$
\left[\begin{array}{cc}
G & K \\
L & 0
\end{array}\right]=\left[\begin{array}{cc}
T^{-1} & 0 \\
0 & I
\end{array}\right]\left[\begin{array}{cc}
\bar{G} & \bar{K} \\
\bar{L} & 0
\end{array}\right] .
$$

PROOF For any system with parameters satisfying (6), we can always find coefficients $\lambda_{i}(i=1, \ldots, v)$, such that $\Omega=\sum_{i=1}^{v} \lambda_{i} \Omega_{i}, \sum_{i=1}^{v} \lambda_{i}=1, \lambda_{i} \geq 0$. If (30) holds for all $i=1, \ldots, v$, take the convex combination of inequalities (30) and then (21) can be obtained. Set $R_{\lambda}=R, S_{\lambda}=S$ and $T_{\lambda}=T$, from Lemma 1, Lemma 2 and Theorem 1, it can be confirmed that the fault detection dynamics (9) are exponentially mean-square stable and satisfy the $H_{\infty}$ attenuation level (12).

Moreover, from Theorem 1, we can confirm that for any uncertain parameters of (1) and (2), the parameters of the fault detection filter can be given by (27). Considering $T_{\lambda}=T$, we can obtain the fault detection filter parameters of the form (31). This ends the proof.

Next, we provide an alternative fault detection filter design result, based on the idea of parameter-dependent stability.

THEOREM 3 Consider networked system (1) with parameter uncertainty (6) and multiple packet dropouts (2). The RNFDFD problem is solvable if there exist 
matrices $\bar{P}_{j}=\bar{P}_{j}^{T}>0, R_{j}, S_{j}, T, \bar{G}, \bar{K}, \bar{L}$ satisfying

$$
\Gamma_{i j}+\Gamma_{j i}<0, \quad 1 \leq i \leq j \leq v
$$

where $\tilde{\rho}_{d}=\operatorname{diag}\left\{\rho_{1} I_{n}, \ldots, \rho_{m} I_{n}\right\}$ and

$$
\Gamma_{i j}=\left[\begin{array}{ccccc}
-I & 0 & 0 & \Sigma_{1} & \Sigma_{2} \\
* & \Sigma_{3} & 0 & \Sigma_{4} & 0 \\
* & * & \Sigma_{5} & \Sigma_{6} & \Sigma_{7} \\
* & * & * & \Sigma_{8} & 0 \\
* & * & * & * & -\gamma^{2} I
\end{array}\right]
$$

with

$$
\begin{aligned}
& \Sigma_{1}=\left[\begin{array}{ll}
0 & \bar{L}
\end{array}\right], \quad \Sigma_{2}=\left[\begin{array}{ll}
0 & -I
\end{array}\right] \\
& \Sigma_{3}=\left[\begin{array}{cc}
\bar{P}_{1 j d}-R_{j d}-R_{j d}^{T} & \bar{P}_{2 j d}-S_{j d}-T_{d} \\
* & \bar{P}_{3 j d}-T_{d}-T_{d}^{T}
\end{array}\right]
\end{aligned}
$$

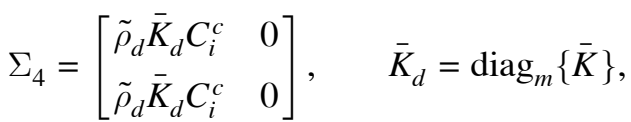

$$
\begin{aligned}
& \Sigma_{5}=\left[\begin{array}{cc}
\bar{P}_{1 j}-R_{j}-R_{j}^{T} & \bar{P}_{2 j}-S_{j}-T \\
* & \bar{P}_{3 j}-T-T^{T}
\end{array}\right] \\
& \Sigma_{6}=\left[\begin{array}{ll}
R_{j}^{T} A_{i}+\bar{K} \sum_{r=1}^{m} \beta^{r} C_{i}^{r} & \bar{G} \\
S_{j}^{T} A_{i}+\bar{K} \sum_{r=1}^{m} \beta^{r} C_{i}^{r} & \bar{G}
\end{array}\right] \\
& \Sigma_{7}=\left[\begin{array}{cc}
R_{j}^{T} B_{i}+\bar{K} D_{i} & R_{j}^{T} E_{i} \\
S_{j}^{T} B_{i}+\bar{K} D_{i} & S_{j}^{T} E_{i}
\end{array}\right] \\
& \Sigma_{8}=-\bar{P}_{j}, \quad \bar{P}_{j}=\left[\begin{array}{cc}
\bar{P}_{1 j} & \bar{P}_{2 j} \\
* & \bar{P}_{3 j}
\end{array}\right] \\
& \bar{P}_{1 j d}=\operatorname{diag}_{m}\left\{\bar{P}_{1 j}\right\}, \quad \bar{P}_{2 j d}=\operatorname{diag}_{m}\left\{\bar{P}_{2 j}\right\} \\
& \bar{P}_{3 j d}=\operatorname{diag}_{m}\left\{\bar{P}_{3 j}\right\}, \quad R_{j d}=\operatorname{diag}_{m}\left\{R_{j}\right\} \\
& S_{j d}=\operatorname{diag}_{m}\left\{S_{j}\right\}, \quad T_{d}=\operatorname{diag}_{m}\{T\} .
\end{aligned}
$$

Moreover, if (32) and (33) are feasible, the parameters of the desired fault detection filter can be given by (31).

Proof Suppose there exist matrices $\bar{P}_{i}=\bar{P}_{i}^{T}>0$, $R_{i}, S_{i}, T, \bar{G}, \bar{K}, \bar{L}$ satisfying (32) and (33). For any system with parameters satisfying (6), one can find coefficients $\lambda_{i}(i=1, \ldots, v)$, such that $\Omega=\sum_{i=1}^{v} \lambda_{i} \Omega_{i}$, $\sum_{i=1}^{v} \lambda_{i}=1, \lambda_{i} \geq 0$. Notice that the following relationship holds

$\sum_{j=1}^{v} \sum_{i=1}^{v} \lambda_{i} \lambda_{j} \Gamma_{i j}=\sum_{i=1}^{v} \lambda_{i}^{2} \Gamma_{i i}+\sum_{i=1}^{v-1} \sum_{j=i+1}^{v} \lambda_{i} \lambda_{j}\left(\Gamma_{i j}+\Gamma_{i j}\right)<0$.

Choosing

$$
R_{\lambda}=\sum_{j=1}^{v} \lambda_{j} R_{j}, \quad S_{\lambda}=\sum_{j=1}^{v} \lambda_{j} S_{j}
$$

and considering the description of the uncertain parameters, one can see that the left-hand side of (21) is equivalent to $\sum_{j=1}^{v} \sum_{i=1}^{v} \lambda_{i} \lambda_{j} \Gamma_{i j}$. Therefore, it follows from (32), (34), and Theorem 1 that the fault detection dynamics (9) are exponentially mean-square stable and satisfy the $H_{\infty}$ attenuation level (12).

The explanation of the fault detection filter parameters of the form (31) is similar to that in the proof of Theorem 2, and is omitted here.

REMARK 3 Based on the idea of parameterdependent stability, Theorem 2 and Theorem 3 provide two different robust fault detection filter design results, both of which are less conservative than parameter-independent results. Since Theorem 1 provides a sufficient and necessary result, all conservatism in Theorem 2 and Theorem 3 reside in 1) the employment of a specific Lyapunov functional and, 2) the treatments in deriving the fault detection design results from Theorem 1. By employing more matrix variables, Theorem 3 provides less conservative results at the costs of more decision variable number and the increment of computational complexity.

If we fix some matrices as invariant for different parameters, we can get a parameter-independent fault detection design result, which is given in the following corollary without proof.

COROLLARY 1 An alternative parameter-independent approach to solve the RNFDFD problem is to impose $P_{1 j}=P_{1}, P_{2 j}=P_{2}, P_{3 j}=P_{3}$ to the conditions (30) indicated in Theorem 2. The parameters of the desired robust fault detection filter can be given in (31).

REMARK 4 It should be pointed out that (30) in Theorem 2 and (32) in Theorem 3 are all LMIs, which can be solved by the efficient interior-point algorithms with the help of Matlab LMI toolbox [2]. Note that if it is not a prior prescribed, the scalar $\gamma^{2}$ can be regarded as an optimization variable. Furthermore, by replacing the feasibility problem with the optimization problem, it is possible to obtain the minimal noise attenuation level bound for the overall fault detection dynamics (9) using Theorem 2 and Theorem 3.

\section{AN ILLUSTRATIVE EXAMPLE}

In this section, an internet-based three-tank system is introduced to illustrate the effectiveness of our proposed fault detection techniques. Three-tank system DTS200 is a nonlinear experimental plant manufactured and provided by Amira Automation Company in Germany. The layout of DTS200 system and all system variables can be found in [24].

The levels of tank $i$ can be measured by pressure sensor $i$, and pump $i$ (set to a constant value) is used to supply water to compensate the outflow of tank $i(i=1,2)$. Unknown input comes from 1$)$ the pump inaccuracy and, 2) the level fluctuation during 
TABLE I

Robust Fault Detection Filter Design Result

\begin{tabular}{|c|c|c|c|}
\hline Method & Theorem 2 & Theorem 3 & Corollary 1 \\
\hline$\gamma$ & 1.0028 & 1.0027 & 1.0031 \\
\hline LMI number & 4 & 10 & 4 \\
\hline Decision variable number & 130 & 184 & 67 \\
\hline $\mathrm{G}\left(\times 10^{-4}\right)$ & {$\left[\begin{array}{ccc}9902 & -41 & 5 \\
-54 & 9897 & 10 \\
-391 & -144 & 9801\end{array}\right]$} & {$\left[\begin{array}{ccc}9901 & -42 & 7 \\
-55 & 9900 & 11 \\
-389 & -146 & 9810\end{array}\right]$} & {$\left[\begin{array}{ccc}9905 & -40 & 5 \\
-51 & 9898 & 10 \\
-362 & -0141 & 9801\end{array}\right]$} \\
\hline $\mathrm{K}\left(\times 10^{-4}\right)$ & {$\left[\begin{array}{cc}-132 & -223 \\
-81 & -253 \\
-602 & -1591\end{array}\right]$} & {$\left[\begin{array}{cc}-131 & -226 \\
-83 & -246 \\
-598 & -1558\end{array}\right]$} & {$\left[\begin{array}{cc}-129 & -214 \\
-77 & -246 \\
-571 & -1522\end{array}\right]$} \\
\hline $\mathrm{L}\left(\times 10^{-9}\right)$ & {$\left[\begin{array}{lll}-1622 & 2832 & -3176\end{array}\right]$} & {$\left[\begin{array}{lll}-1529 & 2425 & -2919\end{array}\right]$} & {$\left[\begin{array}{llll}-1663 & 3095 & -3523\end{array}\right]$} \\
\hline
\end{tabular}

the injection process, which can be regarded as an exponential disturbance and can be mathematically described by adding one term $B w_{k}$ in (1). Consider the potential leak fault $f_{k}$ in tank 3 . The main aim is to alarm the fault after it occurs.

Consider that there are two uncertain parameters (outflow coefficients of tank 1 and tank 2) in the system with their uncertain ranges given by $0.45 \leq$ $a z_{1} \leq 0.55$ and $0.55 \leq a z_{2} \leq 0.65$. In this case, the vertex number of the polytope is 4 . After linearizing the Internet-based three-tank system at the equilibrium point $h_{1}=0.502 \mathrm{~m}, h_{2}=0.218 \mathrm{~m}, h_{3}=0.360 \mathrm{~m}$, discretizing the result with sampling period $T_{s}=0.25 \mathrm{~s}$ and rewriting the system in incremental form, we can obtain the following system parameters.

$$
\begin{aligned}
& A_{1}=\left[\begin{array}{lll}
0.9974 & 0.0000 & 0.0026 \\
0.0000 & 0.9951 & 0.0024 \\
0.0026 & 0.0024 & 0.9950
\end{array}\right] \\
& A_{2}=\left[\begin{array}{lll}
0.9974 & 0.0000 & 0.0026 \\
0.0000 & 0.9955 & 0.0024 \\
0.0026 & 0.0024 & 0.9950
\end{array}\right] \\
& A_{3}=\left[\begin{array}{lll}
0.9979 & 0.0000 & 0.0021 \\
0.0000 & 0.9951 & 0.0024 \\
0.0021 & 0.0024 & 0.9955
\end{array}\right] \\
& A_{4}=\left[\begin{array}{lll}
0.9979 & 0.0000 & 0.0021 \\
0.0000 & 0.9955 & 0.0024 \\
0.0021 & 0.0024 & 0.9955
\end{array}\right] \\
& B_{1}=\left[\begin{array}{lll}
16.2190 & 0.0000 \\
0.0000 & 16.2007 \\
0.0212 & 0.0193
\end{array}\right] \\
& B_{2}=\left[\begin{array}{ll}
16.2190 & 0.0000 \\
0.0000 & 16.2038 \\
0.0212 & 0.0193
\end{array}\right]
\end{aligned}
$$

$$
\begin{aligned}
& B_{3}=\left[\begin{array}{cc}
16.2229 & 0.0000 \\
0.0000 & 16.2007 \\
0.0174 & 0.0193
\end{array}\right] \\
& B_{4}=\left[\begin{array}{cc}
16.2229 & 0.0000 \\
0.0000 & 16.2038 \\
0.0174 & 0.0193
\end{array}\right] \\
& E_{1}=E_{2}=\left[\begin{array}{c}
0.0212 \\
0.0193 \\
16.1997
\end{array}\right] \\
& E_{3}=E_{4}=\left[\begin{array}{c}
0.0174 \\
0.0193 \\
16.2036
\end{array}\right] \\
& C_{1}=C_{2}=C_{3}=C_{4}=\left[\begin{array}{lll}
1 & 0 & 0 \\
0 & 1 & 0
\end{array}\right] \\
& D_{1}=D_{2}=D_{3}=D_{4}=\left[\begin{array}{ll}
0 & 0 \\
0 & 0
\end{array}\right] \text {. }
\end{aligned}
$$

At a certain instant, level heights of tank 1 and tank 2 are packed into different packets and transmitted to fault detection node though different communication channels. Suppose the missing rate for these two sensors is 0.4 and 0.3 , respectively, i.e., $\beta^{1}=0.6, \beta^{2}=0.7$.

First, consider the robust fault detection filter design problem using Theorem 2, Theorem 3 and Corollary 1. The design results are summarized in Table I. From Table I, we can observe that the least conservative robust fault detection filter can be designed by using Theorem 3 . However, Theorem 3 has the most decision variables in the design procedure and is more time consuming. Parameter-independent technique using Corollary 1 is more conservative than the parameter-dependent one using Theorem 2 or Theorem 3, but it can be solved rapidly. The technique in Theorem 2 is a 


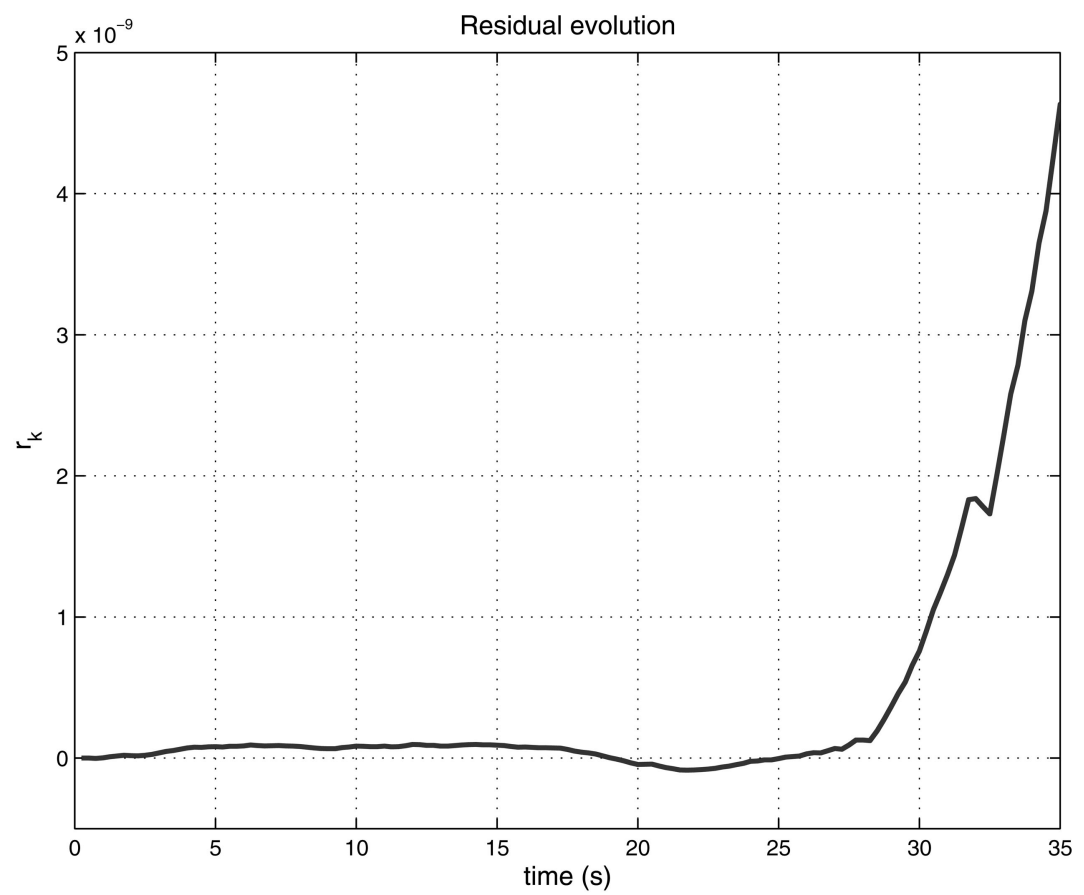

Fig. 2. Robust fault detection filter output $r_{k}$.

TABLE II

Thresholds for Fault Detection

\begin{tabular}{cccc}
\hline \hline Method & Theorem 2 & Theorem 3 & Corollary 1 \\
\hline$J_{t h}\left(\times 10^{-17}\right)$ & 6.3417 & 6.0282 & 7.0395 \\
\hline
\end{tabular}

trade-off between conservatism and computational complexity.

Next, let us determine the thresholds for each robust fault detection filters. For $k=0,1, \ldots, 300$, the unknown input $w_{k}$ is taken as $w_{k}=10^{-6} \times \sin (10 k) \times$ $v_{k}$, where $v_{k}$ is uniformly distributed over $[-0.5,0.5]$. The fault signal is set to be $f_{k}=0$. After 400 times Monte Carlo simulations without fault, the following thresholds shown in Table II can be obtained.

Finally, real time fault detection simulation is provided. The fault signal is set to be $f_{k}=0$ before $t=25 \mathrm{~s}$ and $f_{k}=1$ otherwise. The outflow coefficients of tank 1 and tank 2 are randomly chosen as $a z_{1}=$ 0.4833 and $a z_{2}=0.6$. Three robust fault detection filters designed by the aforementioned approaches have been tested for the time domain simulation. Here, attention is focused on the parameter-dependent case stated in Theorem 3, and the results in other cases are similar. Fig. 2 shows the residual evolution signal of the robust fault detection filter and Fig. 3 provides the evaluation function of the residual signal, where the dashed line is the threshold $J_{t h}$. From Fig. 3, we can observe that the fault can be detected in 9 seconds after its occurrence.

The detection delay steps in all cases are listed in Table III, from which we know that both the parameter-independent result and the parameter-
TABLE III

Fault Detection Time Delay Step $T_{d}$

\begin{tabular}{cccc}
\hline \hline Method & Theorem 2 & Theorem 3 & Corollary 1 \\
\hline$T_{d}(s)$ & 10 & 9 & 11 \\
\hline
\end{tabular}

dependent method can be used for fault detection and generally, the less conservative the fault detection filter, the faster the fault detection process.

\section{CONCLUSION}

In this paper, the robust fault detection problem for a class of networked systems with distributed sensors has been investigated. Data from different sensors are packed in different packets and then transmitted via different communication channels with different characteristics. Since the bandwidth of the network is limited, packets from different channels have different missing rates. A diagonal matrix has been utilized to model this multiple packet dropout and our aim is to design a robust fault detection filter such that, for all incomplete measurements and system uncertainties, the residual of the fault detection filter approaches the fault signal as much as possible. After properly state augmentation, the original problem has been transformed into a robust $H_{\infty}$ filtering problem and two different parameter-dependent results have been proposed in the form of LMIs, which can be easily solved by using the efficient convex optimization method. Simulation results on an internet-based three-tank system have been given to demonstrate the applicability of the proposed design procedures. 


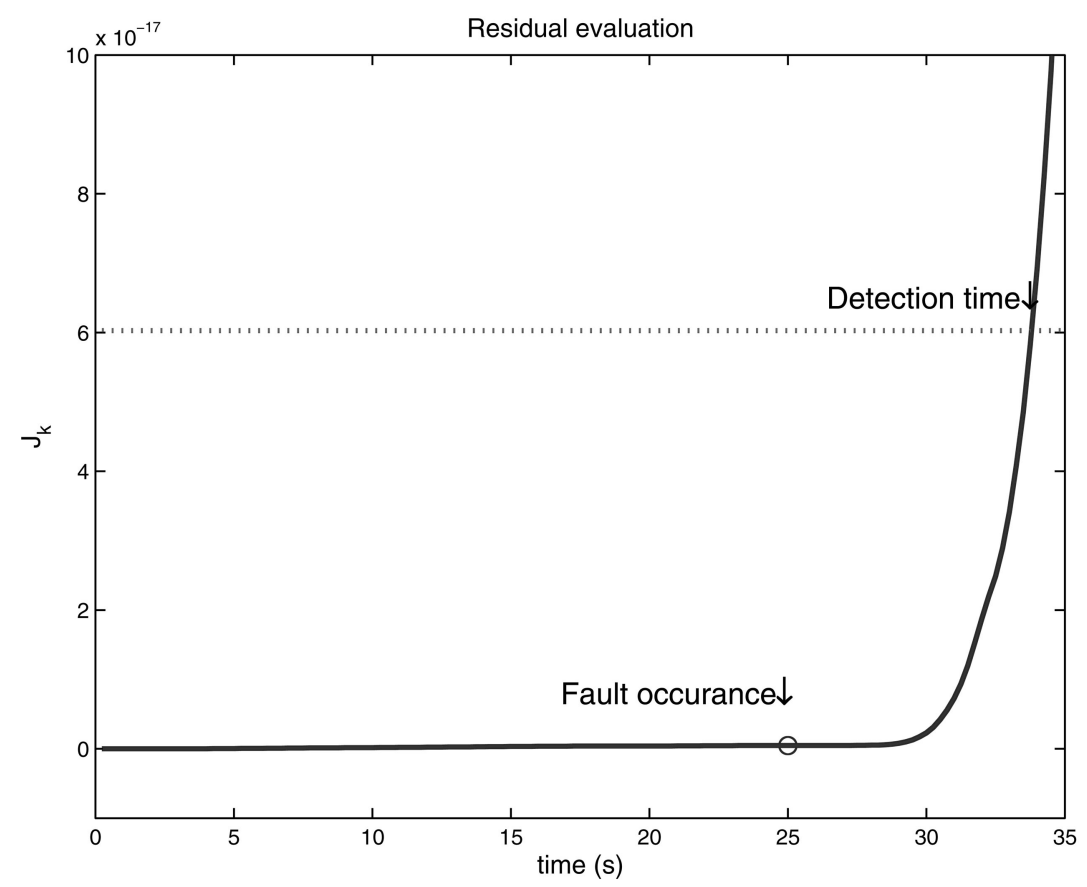

Fig. 3. Residual evaluation function.

\section{REFERENCES}

[1] Azimi-Sadjadi, B.

Stability of networked control systems in the presence of packet losses.

In Proceedings of 42nd IEEE Conference on Decision and Control, Piscataway, NJ, 2003, 676-681.

[2] Boyd, S., Ghaoui, L. E., Feron, E., and Balakrishnan, V. Linear Matrix Inequalities in System and Control Theory. SIAM Studies in Applied Mathematics, Philadelphia, PA, 1994.

[3] Chow, M. Y. and Tipsuwan, Y.

Network-based control systems: A tutorial.

In Proceedings of IECON '01: The 27th Annual Conference of the IEEE Industrial Electronics Society, 2001, 1593-1602.

[4] Ding, S. X., Jeinsch, T., Frank, P. M., and Ding, E. L. A unified approach to the optimization of fault detection systems.

International Journal of Adaptive Control and Signal Processing, 14, 7 (2000), 725-745.

[5] Fang, H., Ye, H., and Zhong, M.

Fault diagnosis of networked control systems. In Proceedings of IFAC SAFEPROCESS, Beijing, 2006, $1-12$.

[6] Feng, G.

Robust $H_{\infty}$ filtering for fuzzy dynamic systems.

IEEE Transactions on Aerospace and Electronic Systems, 41, 2 (2005), 658-670.

[7] Gao, H. and Wang, C.

A delay-dependent approach to robust $H_{\infty}$ filtering for uncertain discrete-time state-delayed systems. IEEE Transactions on Signal Processing, 52, 6 (2004), 1631-1640.

[8] Gao, H. and Chen, T.

Estimation for uncertain systems with limited communication capacity.

IEEE Transactions on Automatic Control, 52, 11 (2007), 2070-2084
[9] He, X., Wang, Z., and Zhou, D.

Robust $H_{\infty}$ filtering for networked systems with multiple state-delays.

International Journal of Control, 80, 8 (2007), 1217-1232.

[10] Hespanha, J. P., Naghshtabrizi, P., and Xu, Y.

A survey of recent results in networked control systems. Proceedings of the IEEE, 95, 1 (2007), 138-162.

[11] Jeong, S. and Tugnait, J.

Multisensor tracking of a maneuvering target in clutter using IMMPDA filtering with simultaneous measurement update.

IEEE Transactions on Aerospace and Electronic Systems, 41, 3 (2005), 1122-1131.

[12] Kaplan, L. M.

Global node selection for localization in a distributed sensor network.

IEEE Transactions on Aerospace and Electronic Systems, 42, 1 (2006), 113-135.

[13] Lian, F. L., Moyne, J., and Tilbury, D. Network design consideration for distributed control systems.

IEEE Transactions on Control Systems Technology, 10, 2 (2002), 297-307.

[14] Liu, G. P., Xia, Y. Q., Chen, J., Rees, D., and Hu, W. S. Networked predictive control of systems with random network delays in both forward and feedback channels. IEEE Transactions on Industrial Electronics, 54, 3 (2007), 1282-1297.

[15] Moges, M. and Robertazzi, T. G.

Wireless sensor networks: Scheduling for measurement and data reporting.

IEEE Transactions on Aerospace and Electronic Systems, 42, 1 (2006), 327-340.

[16] Patton, R. J., Kambhampati, C., Casavola, A., Zhang, P., Ding, S., and Sauter, D.

A generic strategy for fault-tolerance in control systems distributed over a network.

European Journal of Control, 13, 2-3 (2007), 280-296. 
Model validation and state estimation for uncertain continuous-time systems with missing discrete-continuous data.

Computers and Electrical Engineering, 25, 1 (1999), 29-43.

[18] Smith, S. C. and Seiler, P.

Estimation with lossy measurements: Jump estimators for jump systems.

IEEE Transactions on Automatic Control, 48, 12 (2003), 2163-2171.

[19] Sun, S., Xie, L., Xiao, W., and Soh, Y. C.

Optimal linear estimation for systems with multiple packet dropouts.

Automatica, 44, 5 (2008), 1333-1342.

[20] Sun, S., Xie, L., Xiao, W., and Soh, Y. C.

Optimal filtering for systems with multiple packet dropouts.

IEEE Transactions on Circuits and Systems-II, 55, 7 (2008), 695-699.

[21] Walsh, G. C., Hong, Y., and Bushnell, L. G.

Stability analysis of networked control systems.

IEEE Transactions on Control Systems Technology, 10, 1 (2002), 438-446.

[22] Wang, Z., Yang, F., Ho, D. W. C., and Liu, X.

Robust $H 1$ filtering for stochastic time-delay systems with missing measurements.

IEEE Transactions on Signal Processing, 54, 7 (2006), 2579-2587.

[23] Wang, Z., Yang, F., Ho, D. W. C., and Liu, X.

Robust $H_{\infty}$ control for networked systems with random packet losses.

IEEE Transactions on Systems, Man and Cybernetics-Part $B, 37,4$ (2007), 916-924.

[24] Xie, X. Q., Zhou, D. H., and Jin, Y. H.

Strong tracking filter based adaptive generic model control.

Journal of Process Control, 9, 4 (1999), 337-350.
[25] Xu, Y. and Hespanha, J. P.

Estimation under uncontrolled and controlled communications in networked control systems. In Proceedings of 44th IEEE Conference CDC-ECC'05, 2003, 842-847.

[26] Yang, F., Wang, Z., Hung, Y. S., and Shu, H. Mixed $H_{2}=H_{\infty}$ filtering for uncertain systems with regional pole assignment.

IEEE Transactions on Aerospace and Electronic Systems, 42, 2 (2005), 438-448.

[27] Yang, S. H., Dai, C., and Knott, R.

Remote maintenance of control system performance over the Internet.

Control Engineering Practice, 15, 5 (2007), 533-544.

[28] Yu, M., Wang, L., and Chu, T.

Stability analysis of networked systems with packet dropout and transmission delays: Discrete-time case. Asian Journal of Control, 7, 4 (2005), 433-439.

[29] Yue, D., Han, Q. L., and Peng, C.

State feedback controller design for networked control systems.

IEEE Transactions on Circuits and Systems-II, 51, 11 (2004), 640-644.

[30] Yue, D. and Han, Q. L.

Network-based robust $H_{\infty}$ filtering for uncertain linear systems.

IEEE Transactions on Signal Processing, 54, 11 (2006), 4293-4301.

[31] Zhang, P., Ding, S. X., Frank, P. M., and Sader, M. Fault detection of networked control systems with missing measurements.

In Proceedings of Asian Control Conference, Melbourne, Australia, 2004, 1258-1263.

[32] Zhang, W., Branicky, M. S., and Phillips, S. M.

Stability of networked control systems.

IEEE Control Systems Magazine, 21, 1 (2001), 84-99.

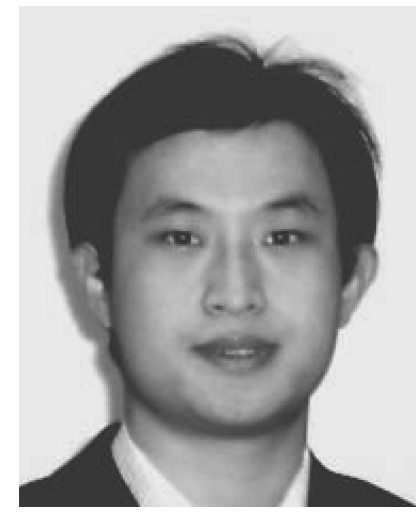

Xiao He received the B.E. degree in electric engineering in 2004 from Beijing Institute of Technology, Beijing, China and the Ph.D. degree in 2010 from Tsinghua University, Beijing, China.

His research interests include robust filtering with incomplete observation, fault diagnosis and isolation, and networked control systems. Mr. He has published around 10 papers in refereed international journals. 


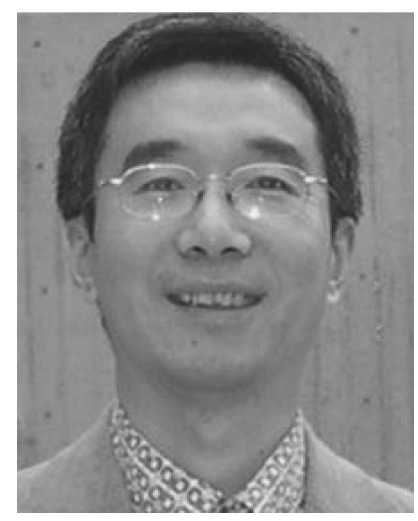

Zidong Wang (SM'03) was born in Jiangsu, China, in 1966. He received the B.Sc. degree in mathematics in 1986 from Suzhou University, Suzhou, China, and the M.Sc. degree in applied mathematics in 1990 and the Ph.D. degree in electrical engineering in 1994, both from Nanjing University of Science and Technology, Nanjing, China.

He is currently Professor of Dynamical Systems and Computing in the Department of Information Systems and Computing, Brunel University, UK. From 1990 to 2002, he held teaching and research appointments in universities in China, Germany, and the UK. He is a holder of the Alexander von Humboldt Research Fellowship of Germany, the JSPS Research Fellowship of Japan, William Mong Visiting Research Fellowship of Hong Kong. His research interests include dynamical systems, signal processing, bioinformatics, control theory and applications.

Dr. Wang has published more than 100 papers in refereed international journals. He serves as an associate editor for 11 international journals, including IEEE Transactions on Automatic Control, IEEE Transactions on Control Systems Technology, IEEE Transactions on Neural Networks, IEEE Transactions on Signal Processing, and IEEE Transactions on Systems, Man, and Cybernetics-Part C. $\mathrm{He}$ is a a Fellow of the Royal Statistical Society and a member of the program committee for many international conferences.

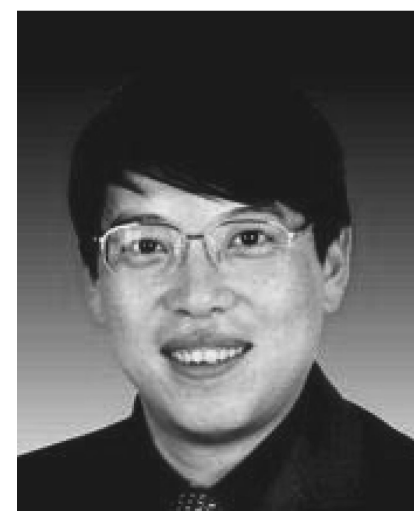

Y. D. Ji was born in Beijing, China, in 1962. He received his B.E. and M.S. degree from the Department of Automation, Tsinghua University, in 1985, and 1989 , respectively.

He is currently an associate professor in the Department of Automation, and Vice President of the Research Institute of Information Technology (RIIT), at Tsinghua University.

Dr. Ji has published widely, with over 40 journal publications in the areas of digital signal process, fault diagnosis, modeling and simulations, and train control system of high speed railway.

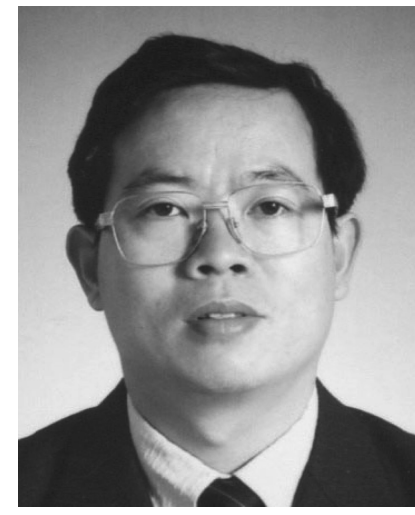

D. H. Zhou (SM'99) received his B.E., M.S. and Ph.D. degrees all from the Department of Electrical Engineering, Shanghai Jiaotong University, in 1985, 1988, and 1990, respectively.

He was an Alexander von Humboldt Research Fellow (1995-1996) in the University of Duisburg, Germany; and a visiting professor at Yale University (July 2001-Jan. 2002). He is currently a professor in the Department of Automation at Tsinghua University.

Dr. Zhou has published widely with over 150 journal publications, and three monographs in the areas of process identification, diagnosis, and control. He serves the profession in many capacities such as in the IFAC Technical Committee on Fault Diagnosis and Safety, as Deputy General Secretary of Chinese Association of Automation (CAA), and as council member of CAA. He was also the NOC Chair of the 6th IFAC Symposium on SAFEPROCESS, 2006. 